\title{
Novel calcineurin A (PPP3CA) variant associated with epilepsy, constitutive enzyme activation and downregulation of protein expression
}

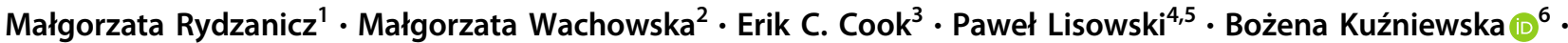 \\ Krystyna Szymańska ${ }^{7}$ Sebastian Diecke ${ }^{5}$ - Alessandro Prigione $\mathbb{1}^{5}{ }^{5} \cdot \mathrm{Krzysztof}$ Szczałuba $\mathbb{1}^{1}$. \\ Aleksandra Szybińska ${ }^{8}$. Agnieszka Koppolu ${ }^{1,9}$ - Victor Murcia Pienkowski ${ }^{1,9}$ • Joanna Kosińska ${ }^{1}$. \\ Małgorzata Wiweger ${ }^{8} \cdot$ Grażyna Kostrzewa $^{1} \cdot$ Małgorzata Brzozowska ${ }^{10} \cdot$ Dorota Domańska-Pakieła $^{11}$.

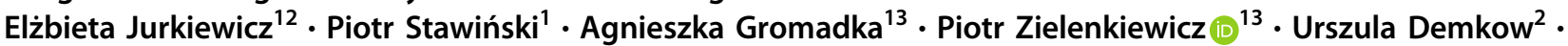

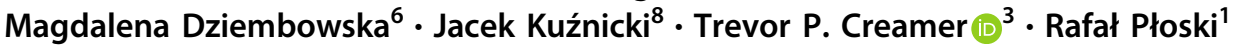

Received: 16 February 2018 / Revised: 22 June 2018 / Accepted: 9 August 2018 / Published online: 25 September 2018

(c) European Society of Human Genetics 2018

\begin{abstract}
PPP3CA encodes calmodulin-binding catalytic subunit of calcineurin, a ubiquitously expressed calcium/calmodulin-regulated protein phosphatase. Recently de novo PPP3CA variants were reported as a cause of disease in 12 subjects presenting with epileptic encephalopathy and dysmorphic features. We describe a boy with similar phenotype and severe early onset epileptic encephalopathy in whom a novel de novo c.1324C $>\mathrm{T}$ (p.(Gln442Ter)) PPP3CA variant was found by whole exome sequencing. Western blot experiments in patient's cells (EBV transformed lymphocytes and neuronal cells derived through reprogramming) indicate that despite normal mRNA abundance the protein expression level is strongly reduced both for the mutated and wild-type protein. By in vitro studies with recombinant protein expressed in $E$. coli we show that c. $1324 \mathrm{C}>\mathrm{T}$ (p. (Gln442Ter)) results in constitutive activation of the enzyme. Our results confirm the role of PPP3CA defects in pathogenesis of a distinct neurodevelopmental disorder including severe epilepsy and dysmorphism and provide further functional clues regarding the pathogenic mechanism.
\end{abstract}

These authors contributed equally: Małgorzata Rydzanicz, Małgorzata Wachowska

Electronic supplementary material The online version of this article (https://doi.org/10.1038/s41431-018-0254-8) contains supplementary material, which is available to authorized users.

Rafał Płoski

rploski@wp.pl

1 Department of Medical Genetics, Medical University of Warsaw, Warsaw, Poland

2 Department of Laboratory Diagnostics and Clinical Immunology of Developmental Age, Medical University of Warsaw, Warsaw, Poland

3 Center for Structural Biology and Department of Molecular \& Cellular Biochemistry, University of Kentucky, Lexington, USA

4 Institute of Genetics and Animal Breeding of the Polish Academy of Sciences, Jastrzębiec, Poland

5 Max-Delbrück-Center for Molecular Medicine (MDC) in the Helmholtz Association, Berlin, Germany

6 Centre of New Technologies, University of Warsaw, Warsaw, Poland

\section{Introduction}

Calcineurin $(\mathrm{CaN})$ is a calcium/calmodulin $(\mathrm{CaM})$-regulated protein phosphatase consisting of a $19-\mathrm{kD}$ calcium-binding calcineurin B subunit (CaNB), and a 61-kD calmodulin-

7 Department of Experimental and Clinical Neuropathology, Mossakowski Medical Research Center, Polish Academy of Sciences, Warsaw, Poland

8 International Institute of Molecular and Cell Biology in Warsaw, Warsaw, Poland

9 Postgraduate School of Molecular Medicine, Medical University of Warsaw, Warsaw, Poland

10 Department of Forensic Medicine, Medical University of Warsaw, Warsaw, Poland

11 Department of Child Neurology and Epileptology, The Children's Memorial Health Institute, Warsaw, Poland

12 Department of Diagnostic Imaging, The Children's Memorial Health Institute, Warsaw, Poland

13 Department of Bioinformatics, Institute of Biochemistry and Biophysics, Polish Academy of Sciences, Warsaw, Poland 
a

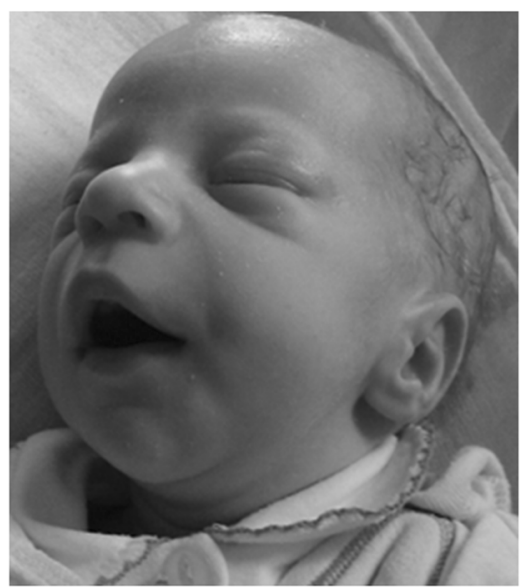

b

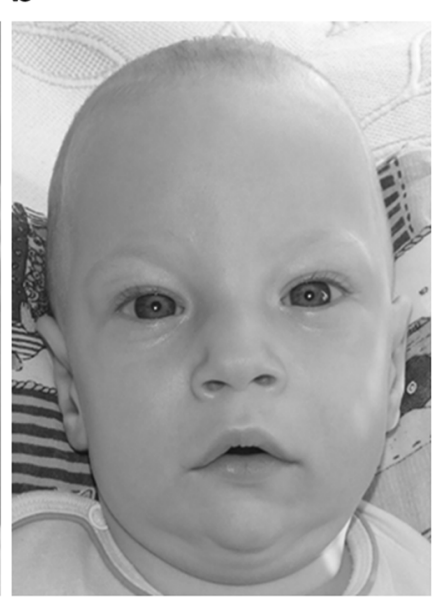

C

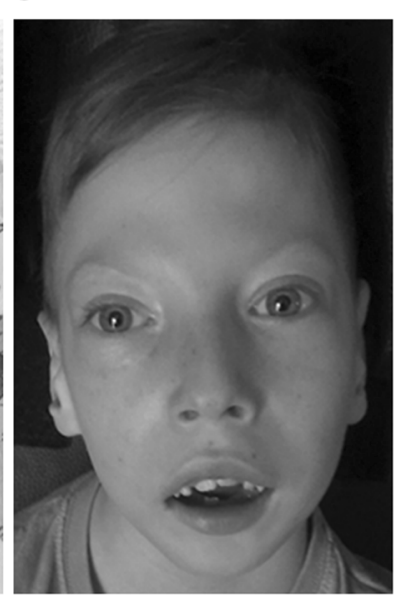

Fig. 1 Facial features of proband. Slight dysmorphia consisting mainly of microretrognathia at age 2 weeks (a), and then evolving into slightly turricephalic skull, hypertelorism, wide nasal alae, relative

binding catalytic subunit, calcineurin A (CaNA) encoded by the PPP3CA gene [1]. CaN is ubiquitously expressed with particularly high abundance in the brain where it controls the phosphorylation states of important synaptic receptors including NMDARs [2] and GABAARs [3]. In particular, $\mathrm{CaN}$ activation was required for the decrease of GABAARs at the synapse in neuronal in vitro model of status epilepticus [3]. Furthermore, $\mathrm{CaN}$ inhibitors have anticonvulsant effects in different rodent epilepsy models [2, 4-6].

Recently de novo PPP3CA variants were described as disease causing in 12 subjects with severe neurodevelopmental disease with seizures and dysmorphy (MIM 617711) [7, 8]. In the study by Myers et al. [7] the link between described variants and the disease was supported by statistical significance of the excess of de novo variants found in PPP3CA after accounting for the length and mutability of the gene as well as the size of the studied cohort $(4,760$ patients). Mizuguchi et al., reported six disease associated PPP 3CA variants, five of these were functionally studied in a yeast model system which showed that variants located within two different domains (catalytic and autoinhibitory) alter protein in distinct ways. For the catalytic variants loss of function was observed, whereas variants in autoinhibitory domain (AID) caused gain of function [8]. Interestingly, it was proposed that the variants altering AID but not those affecting the catalytic domain, were associated with dysmorphy and skeletal anomalies [8].

No functional analyses were reported for regulatory domain (RD) variants leaving unanswered question how exactly these variants cause the disease. Elucidation of the pathomechanism of PPP $3 C A$ related disease is important since $\mathrm{CaN}$ is a molecular target of two potent inhibitors: cyclosporin A and tacrolimus (FK506) which are widely used in clinics for immunosuppression. macrostomia with open mouth and full lips as well as microretrognathia at ages of 6 months (b) and 7 years (c)

Here we describe a boy with severe early onset epileptic encephalopathy in whom a novel de novo c.1324C $>\mathrm{T}$ (p. (Gln442Ter)) PPP3CA variant located in RD was found. Studies using recombinant proteins show that c.1324C $>\mathrm{T}$ (p.(Gln442Ter)) results in the constitutive activation of the enzyme. Moreover, analysis of protein level in patient's cells indicate that despite normal mRNA abundance the protein expression is strongly reduced both for the mutated and wild-type $\mathrm{CaN}$. Our results confirm the role of $P P P 3 C A$ variants in the pathogenesis of epilepsy and provide further functional clues regarding their pathogenic mechanism.

\section{Case report}

\section{Ethical approval}

All procedures performed in this study were in accordance with the ethical standards of the institutional research committee and with the 1964 Helsinki declaration and its later amendments or comparable ethical standards. The study was approved by the ethical committee at the Medical University in Warsaw. Informed consent was obtained from the patient's parents including consent for publication of patient's photographs.

The boy is the second child of healthy, young unrelated parents, with no family history of neurological/neurodevelopmental disorders. He was born at 40 postconceptional weeks after an uneventful third pregnancy with weight $3100 \mathrm{~g}$, length $57 \mathrm{~cm}$, and occipitofrontal circumference 35 $\mathrm{cm}$, with Apgar score of 10 at 1 and $5 \mathrm{~min}$. At age 7 weeks, he developed episodes of apnea treated with phenobarbital. At 2 months of life, he developed multifocal and generalized clonic seizures. Despite treatment the seizures evolved 
Table 1 Proband's EEG results

\begin{tabular}{ll}
\hline Age & EEG results \\
\hline 14 days & $\begin{array}{l}\text { Normal background, trace alternant, sharp waves at right frontal, central, temporal } \\
\text { regions }\end{array}$ \\
2 months & Normal background, sharp waves and spikes at right hemisphere leads \\
4 months & $\begin{array}{l}\text { Normal background, presence of sleep spindles, spikes and spikes and waves } \\
\text { complexes at right temporal, occipital and parietal regions, sporadic spikes at left side }\end{array}$ \\
6 months & Lack of physiological sleep features, SBA pattern with right side preponderance \\
7 months & Lack of physiological sleep features, SBA pattern with right side preponderance \\
7 months 26 days & Lack of physiological sleep features, SBA pattern \\
10 months & Lack of physiological sleep features, SBA pattern with right side preponderance \\
11 months & Lack of physiological sleep features, SBA pattern with right side preponderance \\
1 year 4 months & Lack of physiological sleep features, SBA pattern \\
2 years 10 months & Lack of physiological sleep features, SBA pattern \\
\hline SBA suppression burst activity
\end{tabular}

into polymorphic attacks consisting of: tonic and clonic seizures, epileptic spasms, abnormal eye movements and nystagmus. The number of attacks per day was up to several dozen. Therapeutic trials with phenobarbital, vigabatrin, topiramate, valproate, levetiracetam, lamotriginum, corticosteroids, acetazolamidum, ketogenic diet, pyridoxine, pyridoxal phosphate, and folinic acid failed to control the seizures. Infections of the upper and lower respiratory tract and pneumonia often occurred from the first months of life. At 6 months he was diagnosed with sepsis.

Several dysmorphic features were noted early in life with the phenotype evolving later (see Fig. 1). Since birth dysmorphic features were observed-receding mandible and narrow palate. Atrial septal defect with left-right shunt was diagnosed at 5 months. Muscle tone evolved from hypotonia to hypertonia in the extremities as well as truncal hypotonia. Later on spastic tetraparesis was diagnosed.

At 3 years of age he manifested severe psychomotor retardation: he was not able to sit without support and had lack of speech. Metabolic evaluation was normal (serum chemistry, liver function tests, ammonia, lactate, pyruvate, amino acids in plasma and CSF, urine organic acids and SAICAR, measurement of free carnitine and acylcarnitines in plasma, lizosomal enzymes). Karyotype was normal. The testing for immune deficiencies was made but no abnormalities were found.

At 3 months of age brain MRI was performed revealing widened lateral ventricles. The next MRI at 10 months showed the ventricular dilatation, discrete supratentorial brain atrophy, slightly thinned corpus callosum (Supplementary Figure 1) Slightly hyperintense signal in the central tegmental tract was also seen on diffusion weighted imaging (not shown).

EEG monitoring started from the age of 2 weeks and was continued in 2-18 months intervals. Totally we evaluated ten recordings, the last one at the age of 2 years 10 months (Table 1). Digital EEG equipment (ELMIKO), standardized
10-20 electrodes system with the ECG registration was used. The EEG recordings included more than $45 \mathrm{~min}$ of spontaneous sleep. First EEG was performed at the age of 14 days: age adequate background activity was registered with presence of trace alternant, single sporadic sharp waves were observed in right frontal, central, and temporal regions. On follow up we observed increased sharp waves and spikes activity in the right hemisphere. Suppression burst activity (SBA) with still persistent preponderance to the right side appeared at the age of 6 months. Periods of flattening in SBA showed tendency to extend over time up to $8 \mathrm{~s}$ at the age of 2 years and 10 months. Together with the presence of SBA pattern physiological rhythms were lost.

\section{Materials and methods}

\section{Whole exome sequencing (WES)}

DNA purified from hair follicles of the proband was analyzed by WES (WES was performed only in proband, not in parents or brother). Library was prepared using SureSelect Human All Exon v5 kit (Agilent, Santa Clara, CA, USA) and paired-end sequenced $(2 \times 100 \mathrm{bp})$ on HiSeq 1500 platform (Illumina, San Diego, CA, USA). Bioinformatics analysis of raw data was performed as described previously [9]. In brief, raw data were analyzed with bcl2fastq software (Illumina) to generate reads in fastq format. After the quality control step including adapter trimming and low quality reads removal, reads were aligned to the hg19 reference genome. Identified variants were annotated with functional information, frequency in population including gnomAD (http://gnomad.broadinstitute.org/), EXaC (http:// exac.broadinstitute.org), ESP6500 (http://evs.gs.wa shington.edu/EVS), 1000 genomes (http://www. 1000 genomes.org/), as well as an in-house database of $>1000$ Polish exomes, and known association with clinical 
phenotypes, based on both ClinVar (https://www.ncbi.nlm. nih.gov/clinvar/) and HGMD (http://www.hgmd.cf.ac.uk) databases.

\section{PPP3CA expression analysis}

In order to study the effect of PPP3CA 1324C $>\mathrm{T}$ (p. (Gln442Ter)) variant on mRNA and protein level quantitative real-time PCR and western blotting were performed, respectively. Both, immortalized B-lymphocytes and induced neuronal cells from the proband and control (proband's healthy brother) were used. Detail description of peripheral blood lymphocytes immortalization and culture, human induced pluripotent stem cell (hiPSCs) reprogramming, small molecule neural precursor cells (smNPC) derivation, smNPC culture, immunostaining, RNA extraction and cDNA synthesis, quantitative real-time PCR (qRTPCR), PCR amplification and sequencing of PPP3CA cDNA fragment comprising variant site and western blotting are given in supplementary materials (Supplementary Methods).

\section{Recombinant CaN protein preparation}

pET15b CnA, CnB was obtained from Addgene (plasmid \#11787) and transformed into E. coli BL-21 (DE3) CodonPlus RIL (Agilent Technologies, La Jolla, CA). $\mathrm{CaN} \triangle 442$ was generated by mutating the codon in the calcineurin gene for Q442 to a stop codon using a Q5 sitedirected mutagenesis kit (NEB, Ipswich, MA). pETCaM1, which encodes the wild-type CALM1 gene from $H$. sapiens, was a gift from Anthony Persechini (University of Missouri at Kansas City, Kansas City, MO). E. coli transformed with any of the plasmids mentioned above were grown in terrific broth (TB) medium until an $\mathrm{OD}_{600}$ of 1.2-1.4 was reached and then induced with $1 \mathrm{mM}$ isopropyl $\beta$-D-1thiogalactopyranoside (IPTG) for $3-4 \mathrm{~h}$ at $37^{\circ} \mathrm{C}$. Proteins were extracted from $E$. coli pellets by suspending in $20 \mathrm{mM}$ Tris (pH 7.5), $200 \mathrm{mM} \mathrm{NaCl}, 5 \mathrm{mM} \beta$-mercaptoethanol, 1 $\mathrm{mM}$ PMSF, and $10 \mathrm{mM}$ imidazole and lysing by sonication. Cell debris was removed via centrifugation. $\mathrm{CaN}$ and $\mathrm{CaN} \triangle 442$ were purified by passing the supernatant of the lysed cells over Ni-NTA His-Bind resin (EMD Millipore, Billerica, MA). Eluted protein was further purified using calmodulin (CaM) Sepharose resin (GE Healthcare, Piscataway). CaM was purified from $E$. coli cell lysate using 2trifluoromethyl-10-aminopropyl-sepharose resin (Center for Structural Biology Chemistry Core Facility at the University of Kentucky). All proteins were purified as described previously [10-12].

A phosphorylated calcineurin substrate corresponding to the regulatory subunit (type II) of cAMP-dependent protein kinase, pRII (WGGDLDVPIPGRFDRRV[pS]VAAE), was purchased from Atlantic Peptides (Lewisburg, PA). An exogenous tryptophan was added to the $\mathrm{N}$-terminal end of the pRII peptide to aid in concentration determination by UV/Vis.

\section{Phosphatase assays}

The RII dephosphorylation assay was used to measure wildtype calcineurin and $\mathrm{CaN} \Delta 442$ activity and was performed under the following solution conditions: $40 \mathrm{mM}$ Tris $(\mathrm{pH}$ 7.5), $100 \mathrm{mM} \mathrm{KCl}, 1 \mathrm{mM} \mathrm{CaCl}_{2}, 1 \mathrm{mM}$ DTT, $5 \mathrm{mM} \mathrm{MnCl}_{2}$, and $30 \mathrm{nM}$ of enzyme, at $30^{\circ} \mathrm{C}$. The RII assay was performed as described in its original publication by Enz et al. [13]. The final $\mathrm{CaM}$ concentration was 90 or $0 \mathrm{nM}$. The concentration of pRII was $100,50,25,12.5,6.25,3.125$, or $0 \mu \mathrm{M}$. The reaction incubated for $7 \mathrm{~min}$ before Biomol Green Reagent (Enzo Life Sciences) was added. Phosphate release was determined by measuring the $\mathrm{OD}_{620 \mathrm{~nm}}$ corresponding to the complex of malachite green molybdate and free phosphate.

\section{Results}

\section{De novo truncating variant in in PPP3CA gene found in proband by WES}

WES was performed to the mean depth of $65 \times ; 87 \%$ of target was covered $\min 20 \times, 93 \%$ - min $10 \times$. We found 119,503 variants passing a default quality. These variants were further filtered to include only those with $<1 \%$ minor allele frequency in all tested databases, and to exclude synonymous variants and variants in non-coding DNA apart from those located within 12 bases from exon-intron junction. The final list of 356 variants was screened for known pathogenic mutations listed in HGMD database (HGMD hits) and searched for mutations consistent with various modes of inheritance. All selected variants considered as potentially disease-causing are listed in Supplementary Table 1.

Considering the phenotype and the characteristics of all variants considered as disease-causing we prioritized a nonsense heterozygous variant in the PPP3CA gene (hg19 chr4:g.101953439G>A， NM_000944.4， Supplementary Figure 2a). The variant was absent in GnomAD (http:// gnomad.broadinstitute.org) and our in-house database of $>1000$ Polish exomes. The c.1324C $>\mathrm{T}$ (p.(Gln442Ter)) introduced a premature stop codon into a gene highly intolerant to monoallelic loss of function variants $(\mathrm{pLI}=$ 1.00, http://exac.broadinstitute.org). The premature stop codon was predicted to cause expression of truncated protein named by us $\mathrm{CaN} \Delta 442$. The c.1324C $>\mathrm{T}$ (p. (Gln442Ter)) PPP3CA variant has been submitted to the 
A

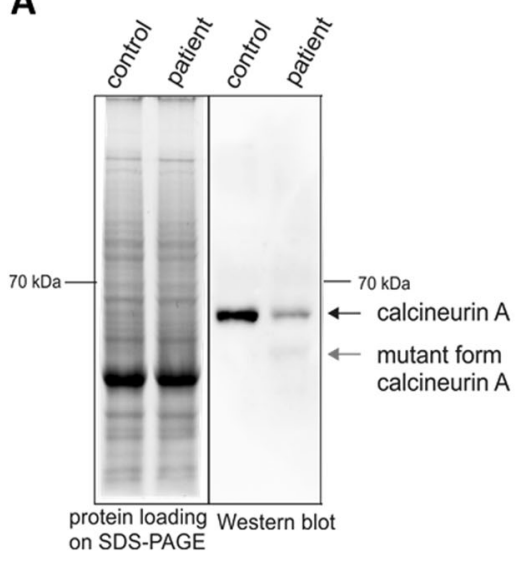

B

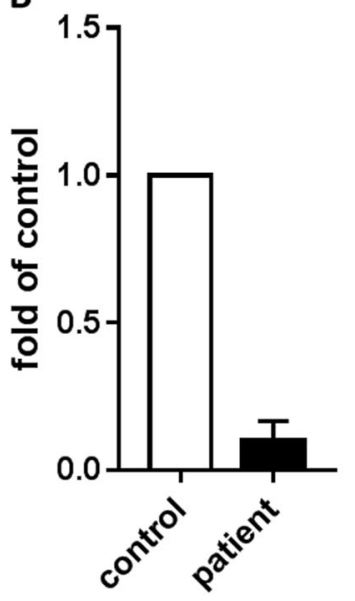

C

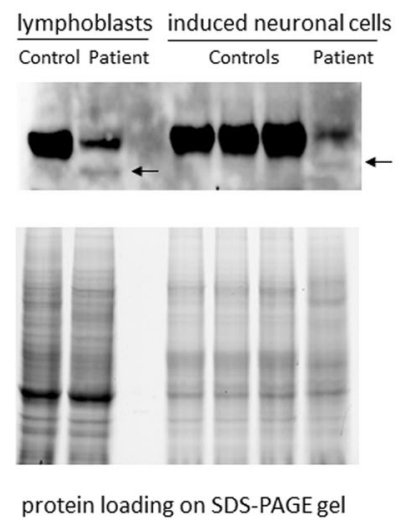

Fig. 2 Analysis of Calcineurin A $\alpha$ protein levels cells from patient with identified variant in PPP3CA gene and control subjects using western blotting (WB). Specific antibody recognizing both normal and mutant form (truncated protein, lacking autoinhibitory domain) of calcineurin A $\alpha$ was used (Abcam \#52761, 1:1000 in 1\% milk). A Representative example of analysis in immortalized B-lymphocytes (left-protein loading shown by TGX stain-free SDS-PAGE gel). B Quantification of WB shown in A done for WT CaN protein, data presented as mean fold change relative to control subject, three independent cultures of each cell line were analyzed. Error bar indicates SEM. C Analysis in induced neuronal cells (lymphoblasts are shown for comparison). Arrows indicate truncated protein.

Leiden Open Variation Database (ID: 00164481, https://da tabases.lovd.nl/shared/individuals/ 00164481).

Using Sanger sequencing we verified presence of c. $1324 \mathrm{C}>\mathrm{T}$ (p. (Gln442Ter))variant in proband and showed that it was absent from both parents and proband's healthy brother, thus indicating a de novo variant (Supplementary Figure 2b,c). Paternity was confirmed with odds $>1,000,000: 1$ using a dedicated forensic STR kit (NGM Select).

We also considered hg 19 chr14:g.091044479G $>$ A in the TTC7B gene (Supplementary Table 1), but Sanger sequencing showed that this variant was present in healthy father and brother of the proband (data not shown).

\section{At mRNA level the allele with CaN 4442 variant and the wild-type (WT) allele are both expressed similarly as in control}

Using deep sequencing of amplicon generated from EBV transformed lymphocytes' cDNA we found that in proband the $\mathrm{CaN} \Delta 442$ variant was present in approximately $50 \%$ of reads indicating that the expression at the mRNA level is comparable to the wild-type variant (Supplementary Figure 3a). We observed that c.1324C $>\mathrm{T}$ (p. (Gln442Ter)) was also expressed in recently described transcripts which contained an additional penultimate exon encoding 11 amino acids (NM_000944, [14]). Out of 155 informative amplicons including this exon $90(58 \%)$ had c.1324C $>\mathrm{T}$ (p.(Gln442Ter)). Thus, we found no evidence of nonsense mediated decay of any of PPP $3 C A$ transcripts with c.1324C $>\mathrm{T}$ (p.(Gln442Ter)) (data not shown).
In parallel, using qRT-PCR we found that total PPP3CA mRNA levels in proband's cells were similar to control's cells (Supplementary Figure 3b).

\section{At the protein level CaN $\Delta 442$ is expressed weakly and is associated with downregulation of the wild type form both in lymphoblasts and neuron-like cells}

Western blot analysis show weak but distinct expression of truncated $\mathrm{CaN} \triangle 442$ protein in proband's lymphoblasts but not in healthy control (Fig. 2a). Unexpectedly, we also observed a strong decrease in expression of WT protein which was confirmed by densitometry (Fig. 2b). The total expressed level of CaN in proband's lymphoblasts was approximately $20 \%$ of the control level. Similar decrease of CaN protein expression including both the mutated and WT variant were observed in proband's neuron-like cells but not in control cells (Fig. 2c). The antibody used for western blot experiments recognized both the truncated and WT protein to a comparable extent as evidenced by experiments with recombinant proteins (Supplementary Figure 4).

\section{CaN $\Delta 442$ mutant protein has activity independent from CaM}

In order to study the functional consequences of $\mathrm{CaN} \Delta 442$ variant we analyzed activity of the mutant vs. WT by an in vitro test using recombinant proteins. Wild-type $\mathrm{CaN}$ is known to require both calcium and CaM for full activation $[1,15,16]$. This is recapitulated in the in vitro RII assay (Table 2 and Fig. 3). We were unable to fit the Michaelis- 
Menten model to the data obtained for $\mathrm{CaN}$ in the absence of CaM, but could do so readily for data collected when $\mathrm{CaM}$ was included. In contrast, $\mathrm{CaN} \Delta 442$ is constitutively active in the absence of CaM (Table 2, Fig. 3). The estimated $V_{\max }$ is essentially identical to that obtained for both WT and mutant $\mathrm{CaN}$ in the presence of $\mathrm{CaM}$, whereas the estimated $K_{\mathrm{m}}$ is slightly higher, suggesting the pRII substrate binds more weakly to $\mathrm{CaN} \Delta 442$ in the absence of CaM.

\section{Discussion}

Using WES we found a novel de novo variant c. $1324 \mathrm{C}>\mathrm{T}$ (p.(Gln442Ter)) in the PPP3CA gene in a patient with epileptic encephalopathy and dysmorphic features. The variant was predicted to lead to the expression of $\mathrm{C}$ terminally truncated protein (CaN $\Delta 442)$. By studying recombinant $\mathrm{CaN} \Delta 442$ we showed that truncated form of $\mathrm{CaN}$ was constitutively activated. We determined experimentally that in patient's cells at the RNA level PPP3CA c.1324C > T (p.(Gln442Ter)) was expressed similarly as WT allele whereas at the protein level its expression was relatively weak. Intriguingly, in two types of cells examined (lymphoblasts and neuron like cells derived by reprogramming) the expression of $\mathrm{CaN} \Delta 442$ was associated with

Table 2 RII phosphatase activity assay analyses for calcineurin and $\mathrm{CaN} \triangle 442$ in the absence and presence of $\mathrm{CaM}$

\begin{tabular}{|c|c|c|c|c|}
\hline & \multicolumn{2}{|l|}{$-\mathrm{CaM}$} & \multicolumn{2}{|l|}{$+\mathrm{CaM}$} \\
\hline & $K_{\mathrm{m}}(\mu \mathrm{M})$ & $\begin{array}{l}V_{\max }(\mu \mathrm{mol} \\
\left.\min ^{-1} \mathrm{mg}^{-1}\right)\end{array}$ & $K_{\mathrm{m}}(\mu \mathrm{M})$ & $\begin{array}{l}V_{\max }(\mu \mathrm{mol} \\
\left.\min ^{-1} \mathrm{mg}^{-1}\right)\end{array}$ \\
\hline $\mathrm{CaN}$ & - & - & $53 \pm 8$ & $0.9 \pm 0.1$ \\
\hline $\mathrm{CaN} \Delta 442$ & $81 \pm 0.3$ & $1.1 \pm 0.4$ & $59 \pm 16$ & $1.2 \pm 0.2$ \\
\hline
\end{tabular}

pronounced downregulation of the full length protein encoded by WT allele.

$\mathrm{CaN}$ is activated in response to increased calcium concentrations in the cell $[1,16]$. The calcium-sensing protein calmodulin (CaM) binds calcium, and can then bind to the regulatory domain (RD; residues 369-470) in the CaNA chain. The CaM binding region within the RD spans residues 391-414. In the absence of CaM, the RD has been shown to be intrinsically disordered [12]. Upon CaM binding, this domain folds, removing an auto inhibitory domain (AID; residues 468-490) from the catalytic site [11, 12]. Removal of the AID is essential for full activation of the wild-type phosphatase.

In the CaN $\Delta 442$ mutant, the RD would be truncated and the AID absent. Accordingly, one may predict that this mutated protein would be active in the absence of CaM. This hypothesis was confirmed by the in vitro RII assays (Table 2 and Fig. 3). Whereas very little activity is observed for WT $\mathrm{CaN}$ in the absence of CaM, CaN $\Delta 442$ is almost as active in the absence as it is in the presence of CaM. In the presence of CaM there is no significant difference in $V_{\max }$ for $\mathrm{CaN}$ and $\mathrm{CaN} \Delta 442$. These data are consistent with the $\mathrm{CaN} \Delta 442$ mutant lacking the AID in the CaNA chain.

In the absence of CaM, the $K_{\mathrm{m}}$ for $\mathrm{CaN} \Delta 442$ is somewhat higher, but the $V_{\max }$ is the same as obtained in the presence of CaM. Previously it was shown that binding of $\mathrm{CaN}$ to pRII is mediated by a substrate binding site located at the interface between CaNA and the CaNB, and that the active site does not contribute significantly to the binding of the pRII peptide $[17,18]$. The pRII binds to a site known as the LxVP substrate binding site, named after the consensus binding sequence. The pRII peptide contains such a binding sequence, and its presence is necessary for binding to $\mathrm{CaN}$. In the absence of $\mathrm{CaM}$, the $\mathrm{RD}$ of $\mathrm{CaN}$ interacts with the LxVP site and may interfere with substrate binding in a competitive manner [19]. This would lead to the weaker $K_{\mathrm{m}}$
Fig. 3 Phosphatase activity for wild-type CaN (solid lines) and $\mathrm{CaN} \Delta 442$ (dashed lines). Kinetics for dephosphorylation of pRII in the a absence and $\mathbf{b}$ presence of $\mathrm{CaM}$
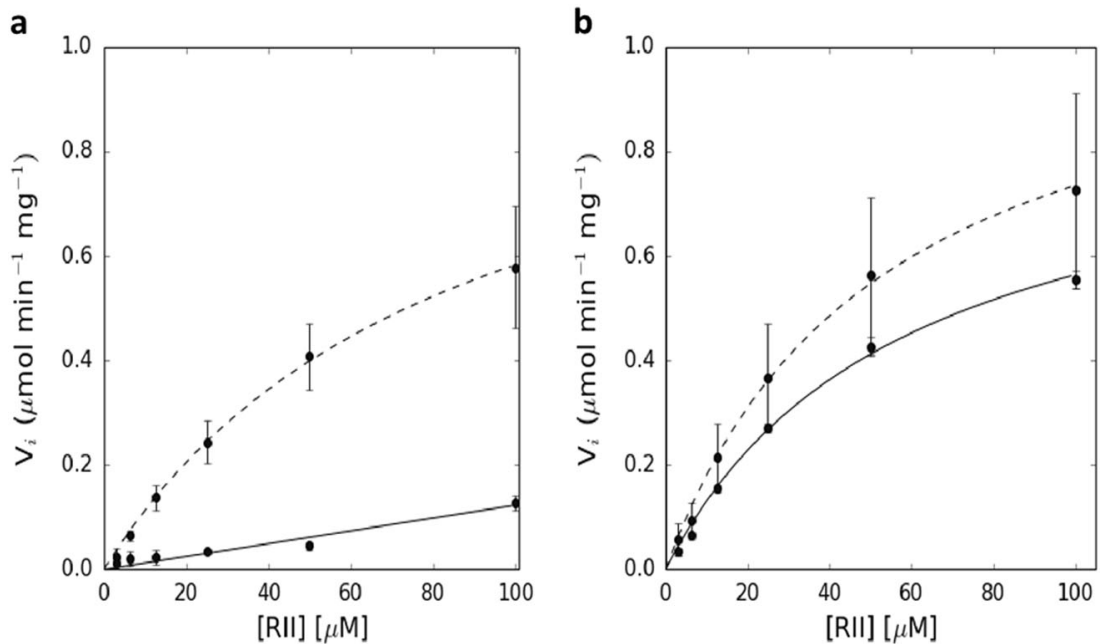
Fig. 4 Structure of human calcineurin, isoform (PDBID 1AUI) [23]. CnA (from $P P P 3 C A)$ is shown in dark blue and $\mathrm{CnB}$ (from $P P P 3 R 1$ ) in grey, with the catalytic iron ion as an orange sphere and the catalytic zinc ion as a pale blue sphere. Calcium ions bound to $\mathrm{CnB}$ are shown as grey spheres. The intrinsically disordered regulatory domain (RD) was generated using MODELLER [24]. The variant identified in this work, c. $1324 \mathrm{C}>\mathrm{T}$ (p. (Gln442Ter)), is shown in red, variants identified by Myers et al. [7] and Deciphering Developmental Disorders Study [20] are purple, those identified by Mizuguchi et al. [8] in yellow, and c.275A $>\mathrm{G}$ (p. (His92Arg)), reported by both the latter two groups, in green. The top inset shows variants that occur in the autoinhibitory domain (AID) and the bottom inset shows those clustered around the active site

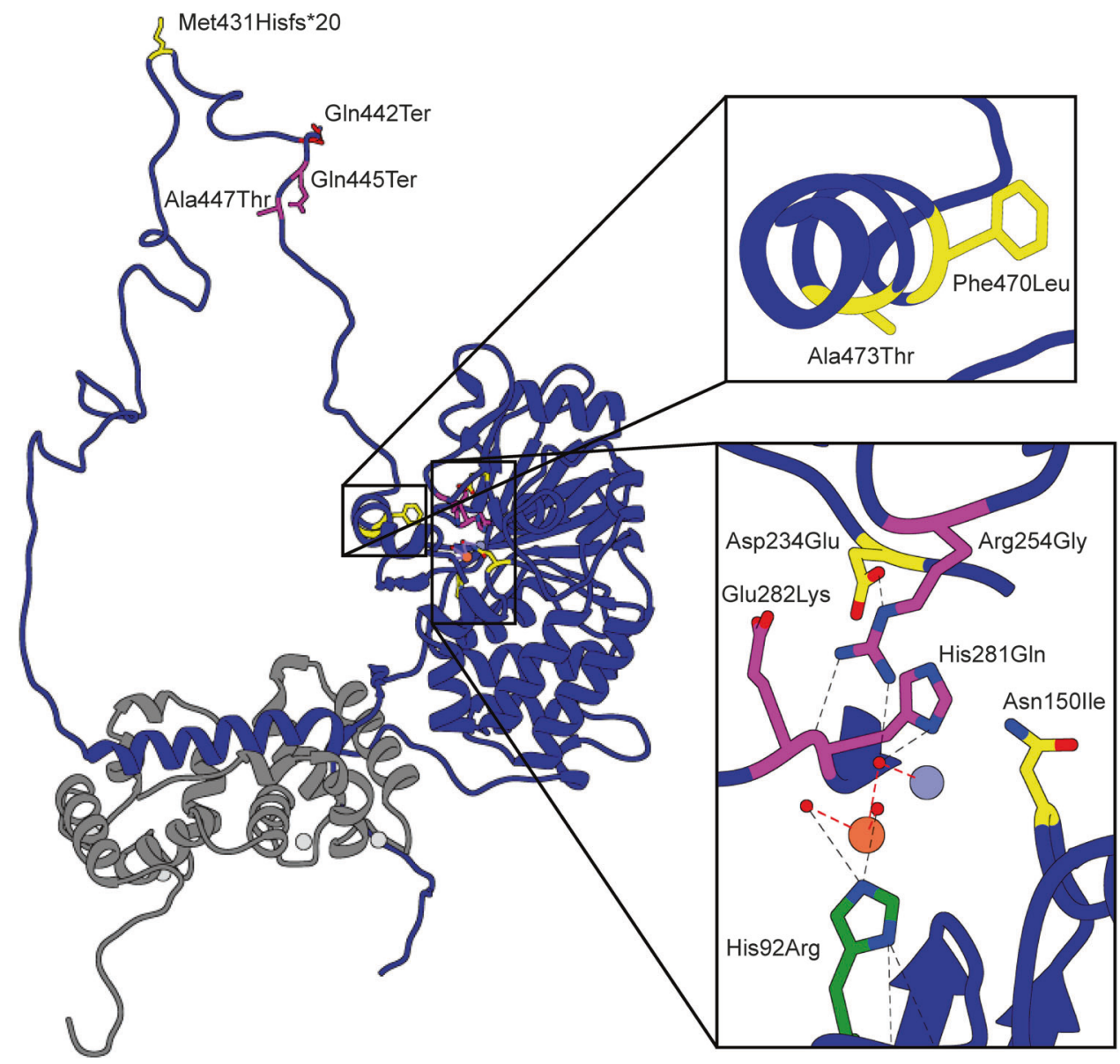

observed for $\mathrm{CaN} \Delta 442$ in the absence of CaM as compared to the presence. When CaM is bound to the RD of CaN, that domain is folded and no longer interacts with the LxVP substrate binding site.

Including the c.1324C $>\mathrm{T}$ (p.(Gln442Ter)) variant discussed in this work, a total of 12 PPP3CA (NM_000944.4) variants have recently been identified [7, 8, 20]. All of these variants were identified in patients with significant abnormalities such as developmental delays, hypotonia and/ or epileptic encephalopathy. The variants are mapped onto a crystal structure of human calcineurin in Fig. 4 and can be seen to be clustered in three distinct locations. The c. $275 \mathrm{~A}>\mathrm{G} \quad$ (p.(His92Arg)), c.449A $>\mathrm{T} \quad$ (p.(Asn150Ile)), c.702C $>$ G (p.(Asp234Glu)), c.760A $>$ G (p. (Arg254Gly)), c.843C > G (p.(His281Gln)) and c.844G >A (p.(Glu282Lys)) are clustered in or around the active site in the catalytic domain. The c.1290dupC (p.(Met431Hisfs*20)), c.1324C $>$ T (p.(Gln442Ter)), c.1333C $>$ T (p.(Gln445Ter)) and c.1339G $>$ A (p.(Ala447Thr)) are located in the RD. Finally, c.1408T >C (p.(Phe470Leu)) and c.1417G >A (p. (Ala473Thr)) are both within the AID. Mizuguchi et al. [8] carried out functional studies on five variants, using $S$. pombe as a model system. Three catalytic domain variants, c.275A $>$ G (p.(His92Arg)), c.449A >T (p.(Asn150Ile)),
c.702C >G (p.(Asp234Glu)), all exhibited loss of function, whereas the two AID variants examined appeared to function at wild-type levels. These observations suggest that the six catalytic domain variants likely lead to loss of function due to disruption of the calcineurin active site. It is less clear how the AID variants lead to pathogenesis. Mizuguchi et al. [8] hypothesized that this could be due to gain of function. If these variants disrupt binding of the AID to the catalytic domain, this could result in constitutively active forms of calcineurin and hence a gain of function.

Until this work, nothing was known about the functional consequences of the $\mathrm{RD}$ variants. The $\mathrm{RD}$ of calcineurin is known to be intrinsically disordered, and yet plays an essential role in activation of the phosphatase [11, 12, 21]. In response to rising calcium levels, calmodulin binds to the $\mathrm{RD}$, leading to a conformational change that removes the AID from the active site, leading to activation. This conformational change appears to involve both the calmodulin binding region within the RD (Ala391 to Arg414) and the formation of a distal alpha-helix between Lys441 and Ile459 [11, 12]. Disruption of this distal helix greatly reduces calcineurin activity, likely a result of the AID remaining at least partially bound in the active site. All of the calcineurin variants within the $\mathrm{RD}$ are $\mathrm{C}$-terminal to the 
calmodulin binding site and would not disrupt its binding (Fig. 4). Two of these variants c.1324C >T (p.(Gln442Ter)) and c. $1333 \mathrm{C}>\mathrm{T}$ (p.(Gln445Ter)), truncate the enzyme such that the AID is no longer present. This could lead to constitutively active forms of the enzyme, as demonstrated here in vitro for the c.1324C>T (p. (Gln442Ter)) variant. The c.1290dupC (p.(Met431Hisfs*20)) variant reported by Mizuguchi et al. [8] would also lack a functional AID and could be constitutively active. The c.1339G>A (p. (Ala447Thr)) variant reported by Myers et al. [7] lies within the distal helix region. A p.Ala447Glu variant that was generated to disrupt distal helix formation was shown to be only partially active in vitro [11]. This latter observation suggests that the c.1339G $>A$ (p.(Ala447Thr)) variant, should it disrupt distal helix formation, would also have reduced activity.

Mizuguchi et al. [8] have suggested that the known calcineurin catalytic domain variants can be classified as loss-of-function and that the two AID variants as gain-offunction. Based on analysis of the c.1324C>T (p. (Gln442Ter)) variant and consideration of existing structure/function information, it would appear that the four RD variants can be parsed into the same two groups. Should they survive nonsense-mediated decay, the c.1324C $>\mathrm{T}$ (p. (Gln442Ter)), c.1333C > T (p.(Gln445Ter)) and c.1290dupC (p.(Met431Hisfs*20)) variants likely lead to gain-of-function, whereas the c.1339G $>\mathrm{A}$ (p.(Ala447Thr)) variant is hypothesized to lead to loss-of-function. How these two classes of variants lead to overlapping sets of pathologies observed in patients is not clear.

Intriguingly, strong downregulation of WT PPP3CA in our proband indicates that constitutive activation of $\mathrm{CaN}$ may induce a decrease in the concentration of this phosphatase which ultimately leads to function deficiency. The downregulation of the PPP3CA protein was not accompanied by decrease of mRNA, either total or transcribed from the variant allele, which emphasizes the importance of studying the protein levels directly instead of relying on transcript level expression. The normal levels of mRNA and lack of nonsense mediated decay of the variant transcripts, including the brain enriched NM_000944 with the additional penultimate exon, have also been found by Mizuguchi et al. for the c.1290dupC, (p. (Met431Hisfs*20)) variant which is predicted to cause a similar PPP3CA truncation as in our proband [8].

It is not clear what cellular regulatory mechanism could be responsible for decreased PPP3CA expression in the presence of normal mRNA levels. One possibility might include recently proposed regulatory pathway involving miRNA 30 [22]. Whereas this prediction should be experimentally confirmed our observation of strongly reduced WT PPPCA protein level could provide a framework to explain the similar disease associated with $P P P 3 C A$ variants with apparently opposing (loss of function vs constitutive activity) effects.

Whereas the study by Mizuguchi et al. [8] and our analyses strongly support functional dichotomy among the disease associated PPP3CA variants the proposed genotype-phenotype correlation is not fully consistent with our findings. In particular, our finding that c.1324C $>\mathrm{T}$ (p. (Gln442Ter)) causes gain of PPP3CA function implies that this and similar variants truncating the AID should cause dysmorphy. Whereas this is the case in our proband it does not hold for c.1333C > T (p.(Gln445Ter)) and c.1290dupC (p.(Met431Hisfs*20)) which were found, respectively, by Myers et al. (pat. 1) and Mizuguchi et al. (pat. 4) in individulas without dysmorphy [7,8]. Thus, more patients are needed to delineate genotype/phenotype correlation in the PPP3CA disease.

In conclusion, our data expand the knowledge on genetics of human epilepsy by describing the clinical phenotype and functional characteristics of a novel PPP3CA variant.

Acknowledgements We thank Dr. Ralf Kühn (Max-Delbrück-Centrum für Molekulare Medizin) and Dr. Heiko Lickert (Helmholtz Center Munich) for kindly providing the control iPSC line XM001. This work was supported by the National Science Centre (NCN) Poland, Grants 2013/11/B/NZ7/04944 (RP), 2014/13/B/NZ3/02947 (MDz), and 2016/22/M/NZ2/00548 (PL).

\section{Compliance with ethical standards}

Conflict of interest The authors declare that they have no conflict of interest.

\section{References}

1. Rusnak F, Mertz P. Calcineurin: form and function. Physiol Rev. 2000;80:1483-521.

2. Wen Y, Fu P, Wu K, Si K, Xie Y, Dan W, et al. Inhibition of Calcineurin A by FK506 Suppresses Seizures and Reduces the Expression of GluN2B in Membrane Fraction. Neurochem Res. 2017;42: 2154-66

3. Eckel R, Szulc B, Walker MC, Kittler JT. Activation of calcineurin underlies altered trafficking of alpha2 subunit containing GABAA receptors during prolonged epileptiform activity. Neuropharmacology. 2015;88:82-90.

4. Freire-Cobo C, Sierra-Paredes G, Freire M, Sierra-Marcuno G. The calcineurin inhibitor Ascomicin interferes with the early stage of the epileptogenic process induced by Latrunculin A microperfusion in rat hippocampus. J Neuroimmune Pharmacol. 2014;9:654-67.

5. Sierra-Paredes G, Sierra-Marcuno G. Ascomycin and FK506: pharmacology and therapeutic potential as anticonvulsants and neuroprotectants. CNS Neurosci Ther. 2008;14:36-46.

6. Kipanyula MJ, Kimaro WH, Seke Etet PF. The Emerging Roles of the Calcineurin-Nuclear Factor of Activated T-Lymphocytes Pathway in Nervous System Functions and Diseases. J Aging Res. 2016;2016:5081021.

7. Myers CT, Stong N, Mountier EI, Helbig KL, Freytag S, Sullivan JE, et al. De Novo Mutations in PPP3CA Cause Severe Neurodevelopmental Disease with Seizures. Am J Hum Genet. 2017;101(4):516-24. 
8. Mizuguchi T, Nakashima M, Kato M, Okamoto N, Kurahashi H, Ekhilevitch $\mathrm{N}$, et al. Loss-of-function and gain-of-function mutations in PPP3CA cause two distinct disorders. Hum Mol Genet. 2018;27:1421-33

9. Ploski R, Pollak A, Muller S, Franaszczyk M, Michalak E, Kosinska J, et al. Does p.Q247X in TRIM63 cause human hypertrophic cardiomyopathy? Circ Res. 2014;114:e2-5.

10. Cook EC, Creamer TP. Calcineurin in a Crowded World. Biochemistry. 2016;55:3092-101.

11. Dunlap TB, Cook EC, Rumi-Masante J, Arvin HG, Lester TE, Creamer TP. The distal helix in the regulatory domain of calcineurin is important for domain stability and enzyme function. Biochemistry. 2013;52:8643-51.

12. Rumi-Masante J, Rusinga FI, Lester TE, Dunlap TB, Williams TD, Dunker AK, et al. Structural basis for activation of calcineurin by calmodulin. J Mol Biol. 2012;415:307-17.

13. Enz A, Shapiro G, Chappuis A, Dattler A. Nonradioactive assay for protein phosphatase $2 \mathrm{~B}$ (calcineurin) activity using a partial sequence of the subunit of cAMP-dependent protein kinase as substrate. Anal Biochem. 1994;216:147-53.

14. Chiocco MJ, Zhu X, Walther D, Pletnikova O, Troncoso JC, Uhl $\mathrm{GR}$, et al. Fine mapping of calcineurin (PPP3CA) gene reveals novel alternative splicing patterns, association of 5'UTR trinucleotide repeat with addiction vulnerability, and differential isoform expression in Alzheimer's disease. Subst Use Misuse. 2010;45:1809-26.

15. Creamer TP. Transient disorder: Calcineurin as an example. Intrinsically Disord Proteins. 2013;1:e26412.
16. Klee CB, Ren H, Wang X. Regulation of the calmodulin-stimulated protein phosphatase, calcineurin. J Biol Chem. 1998;273:13367-70.

17. Grigoriu S, Bond R, Cossio P, Chen JA, Ly N, Hummer G, et al. The molecular mechanism of substrate engagement and immunosuppressant inhibition of calcineurin. PLoS Biol. 2013;11: e1001492.

18. Roy J, Li H, Hogan PG, Cyert MS. A conserved docking site modulates substrate affinity for calcineurin, signaling output, and in vivo function. Mol Cell. 2007;25:889-901.

19. Li SJ, Wang J, Ma L, Lu C, Wang J, Wu JW, et al. Cooperative autoinhibition and multi-level activation mechanisms of calcineurin. Cell Res. 2016;26:336-49.

20. Deciphering Developmental Disorders S. Prevalence and architecture of de novo mutations in developmental disorders. Nature. 2017;542:433-8.

21. Dunlap TB, Guo HF, Cook EC, Holbrook E, Rumi-Masante J, Lester TE, et al. Stoichiometry of the calcineurin regulatory domain-calmodulin complex. Biochemistry. 2014;53:5779-90.

22. Wu J, Zheng C, Wang X, Yun S, Zhao Y, Liu L, et al. MicroRNA-30 family members regulate calcium/calcineurin signaling in podocytes. J Clin Invest. 2015;125:4091-106.

23. Kissinger CR, Parge HE, Knighton DR, Lewis CT, Pelletier LA, Tempczyk A, et al. Crystal structures of human calcineurin and the human FKBP12-FK506-calcineurin complex. Nature. 1995;378:641-4.

24. Sali A, Blundell TL. Comparative protein modelling by satisfaction of spatial restraints. J Mol Biol. 1993;234:779-815 